\title{
Finite Element Analysis of a Tire Steady Rolling on the Drum and Comparison with Experiment
}

\author{
Nikola Korunović* - Miroslav Trajanović - Miloš Stojković - Dragan Mišić - Jelena Milovanović \\ University of Niš, Faculty of Mechanical Engineering, Serbia
}

A finite element (FE) model for analysis of tire rolling on the drum, based on a specially developed $C A D$ model, is presented in the paper. All the changes performed on the geometry of CAD model are automatically propagated to FE model. This makes the FE model very suitable for parametric studies, which help tire designer to quickly find the optimal values of tire design parameters. In this way the tire design process is shortened and the quality of resulting tires improved. The results of finite element analyses conducted on the model have directly been compared to experimental ones, confirming model validity. Equipment and methods used for experimental determination of braking and cornering characteristics of the tire as well as for experimental determination of friction coefficient of tire tread have been shown. The difference between experimental and numerical results was smaller after the calibration of friction coefficient had been performed and in such a way a further improvement of the existing model was achieved.

(C)2011 Journal of Mechanical Engineering. All rights reserved.

Keywords: finite element analysis (FEA), tire design, steady-state rolling (SSR), cornering, braking, parametric

\section{INTRODUCTION}

Due to efficiency in prediction of cornering and braking behavior of tires and moderate requests towards computing resources, steadystate rolling analysis (SSRA) using the finite element method, based on the mixed Eulerian / Lagrangian approach, is often deployed for tire rolling simulations. While purely Lagrangian approach may also be used to simulate the response of a steady rolling tire [1], it is not as efficient and accurate as the mixed one. Explicit FEA, which is a must for dynamic analysis where transients play a significant role, may also be used for tire analysis, but it is neither essential nor efficient for the analysis of a steady rolling tire. While the results of SSRA and explicit analyses may be very similar and in good correlation with the experimental ones, SSRA generally takes a considerably less time to finish [2]. The reasons for such a difference have been found in the smaller mesh size and fewer analysis increments needed for SSRA. In SSRA, finite element (FE) mesh may be refined only in the vicinity of contact area, thanks to the nature of FE formulation.

SSRA of tires yields various kinds of results that may efficiently be used in tire design. Such results can be directly compared to experimental ones, obtained by laboratory testing or testing on specialized vehicles. Typical results of SSRA include the following functions: driving force in terms of angular velocity, cornering force in terms of slip angle or self-aligning torque in term of slip angle. Tire construction parameters like belt width, belt angle or number of plies and operating parameters like inflation pressure, tire load or camber angle may be varied in order to reach the optimal design. Examples of SSRA of tires and parametric studies in which it has been used may be found in [3] to [5].

Finite element analysis (FEA) is generally performed on tires rolling over flat surface, in order to closely simulate their behavior during service. FEA results may directly be compared with experimental ones, obtained using flat bad or flat surface tire testing machines [6] and [1], or specialized vehicles. However, such equipment is space consuming and expensive and is generally hosted by large tire manufacturers or specialized laboratories. Characteristics of a rolling tire may also be evaluated on drum machines [1], [6] and [7] which require less space and are generally more affordable. When such machines are used, a certain level of approximation in footprint geometry is introduced, which becomes more significant as the drum diameter gets smaller. It is 
also difficult to apply a realistic road surface to the drum, because of its curvature. Nevertheless, it is assumed that if tire rolling behavior is successfully modeled and verified on one kind of surface for a range of operating parameters, it may also be used to predict its behavior in different road conditions [1]. In order to compare the results of tire FEA with drum machine testing results, the finite element model has to be modified, to include the definitions of drum surface and drum rotation.

In SSRA of tires special attention should be given to friction modeling, as it has a significant influence on the accuracy of the results. The nature of rubber friction has still not been properly explored. In practice, phenomenological friction models are often used, where friction coefficient of the Coulomb model is defined as a function of sliding velocity, contact pressure and (optionally) of temperature [8]. These models rely on experimental data, obtained using various kinds of equipment intended for sliding of small rubber specimens over artificial road surface [8] to [10].

Previous work of the authors, described in [11] to [14], covers the areas of parametric and knowledge-based tire design, rubber modeling for tire FEA, static analysis and SSRA of tires. This paper describes the use of the methodology presented in [14] for an analysis of the tire rolling on the drum. The purpose of the analysis was to validate the finite element model by comparing FEA results with experimental ones, which were obtained using test rig described in [7] and [10].

A short description of finite element models is given first, followed by a description of equipment and procedures used for braking and cornering tests as well as for determination of tire tread friction coefficient. Then, the results of SSRA, a comparison with the experiment, calibration of friction coefficient and discussion are given, followed by concluding remarks.

\section{FINITE ELEMENT MODELS}

Finite element tire models described in this paper are highly similar to the ones depicted in [14]. The main feature of new models is that the rolling surface is not flat but cylindrical, so that rolling on a drum may be simulated. Hence, only the main features of FE models will be described here, especially their differences compared to [14].

Two FE models are used for the analyses, which are performed in FE code ABAQUS according to algorithm given in [14]. An axisymmetric model is used mainly as a starting point for 3D model creation and for tire inflation analysis, while 3D model is used for inflation, static footprint, acceleration-to-braking and cornering analyses. The models are based on the CAD model developed by the authors (Fig. 1). In adition to basic geometry of tire profile, the CAD model contains a parameterized network of lines and points. Following dimensional changes of tire profile and its structural components, lines and points allocate accordingly, to form the basis for mapped FE mesh. Axisymmetric FE model needs to be created in FE preprocessor only once, before the first of the analyses, using geometry entities exported from the CAD model as described in [14]. For any subsequent design change, the following procedure is deployed: design parameters are changed inside the CAD model; the group of newly positioned points is exported to a neutral format and their spatial position is mapped to the position of nodes of FE model. Mapping and translation of points to nodes is done using a translation program, which is especially written for this purpose. In such a way, it becomes possible to explore a large number of design variations in a very short time, i.e. to conduct parametric studies of tire design. FE model described here may be used for all tire types based on the profile that is constructed in the same or similar manner. If an essentially different type of tire is to be analyzed, a new CAD model needs to be created. This process is a relatively simple one, based on a predefined procedure.

Finite elements of axisymmetric model are grouped accordingly in order to represent the purely rubber components of tire, like sidewall, tread, or bead filler. Composite structural components of the tire, carcass and belts, are created by embedding of surface elements in volume ones. Inside of surface elements rebar layers are defined, which represent steel or rayon cords. The definition of one rebar layer contains a cord area, the distance between the cords, a cord angle and cord material. Bead wire is modeled as isotropic material and the rim is substituted by 
rigid supports. Rubber components are described using Mooney-Rivlin form, where material coefficients are taken from earlier FE models [12]. Steel and rayon have been modeled as linearly elastic materials.

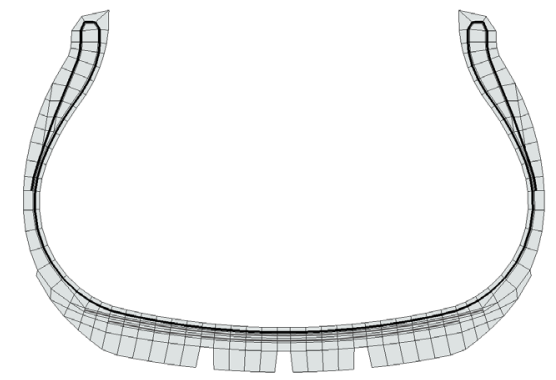

Fig. 1. 2D parametric CAD model of an existing 165/70 R13 tire

Types of finite elements that have been used include axisymmetric hybrid elements with twist for purely rubber structural components, axisymmetric surface elements with a twist for modeling of carcass and belts and axisymmetric solid elements for modeling of the bead wire.

3D FE tire model (Fig. 2) is obtained by rotation of axisymmetric model around tire axis. Dense mesh is created only in the vicinity of tire footprint, as described in [14].

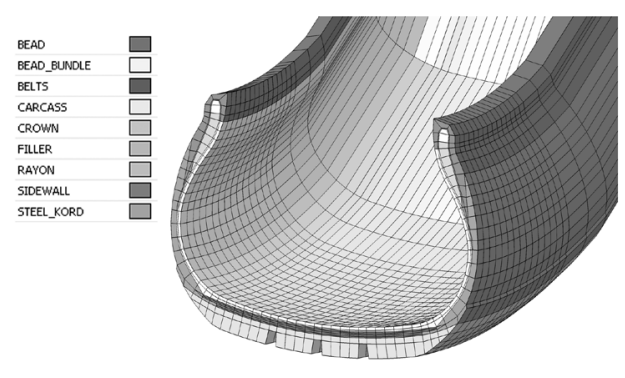

Fig. 2. 3D tire model

3D finite elements, which represent the equivalents to the corresponding axisymmetric ones, are created during the rotation of axisymmetric model. In this way, the 3D FE model is composed of: 8 or 6-noded hybrid elements that represent rubber, 4-noded surface elements for modeling of carcass and 8-noded solid elements used to model the bead wire.

Axisymmetric model contains 473 nodes and 403 elements, while the 3D model contains
21712 nodes and 18538 elements. The numbers of active degrees of freedom are 934 and 63480, respectively.

The drum is modeled as a rigid surface (Fig. 3) and friction between the drum and tread surface is defined using Coulumb model with viscous stick formulation. The variable coefficient of friction is obtained by testing of rubber specimens on Mini- $\mu-$ road [10], as described in the next chapter.

Using the 3D FE model, the following analyses have been conducted: inflation analysis, static footprint analysis, straight line rolling under the action of driving or breaking torque, straight line rolling in fine increments to find the angular velocity of free rolling and cornering analysis at free-rolling.

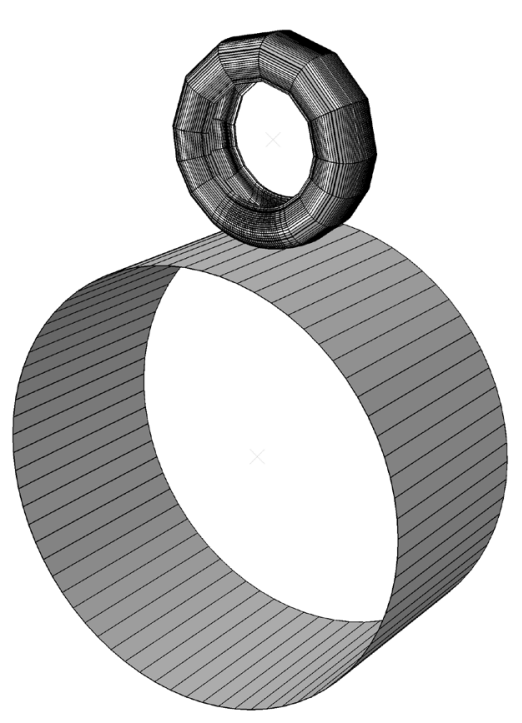

Fig. 3. 3D tire model for FEA of the tire rolling on the drum; diameter of cylindrical surface is equal to diameter of the drum

\section{EXPERIMENTAL SET-UP AND \\ PROCEDURES FOR TIRE BRAKING AND \\ CORNERING AND RUBBER FRICTION TESTING}

\subsection{Tire Testing Rig}

In order to test tire behavior during braking and cornering, dynamic tire force measuring device [7] (Fig. 4), set up at Helsinki University of Technology, Laboratory of Automotive 
Engineering, has been used. The diameter of machine's drum, which is equipped with the dynamometer, equals to $1219 \mathrm{~mm}$. Maximal circumferential speed at which reasonable results may be obtained is around $150 \mathrm{~km} / \mathrm{h}$. The drum is coated with a layer of safety walk paper. Wheel load can be adjusted up to the maximal value of $5000 \mathrm{~N}$. The slip angle can be adjusted during measuring process, in the range of -10 to $+10^{\circ}$. Camber angle may vary from -4 to $+4^{\circ}$. All six forces and moments acting between the tire and the drum, as defined in [6] and [14], may be measured, as well as the rotational speed of the tire. The tire, photographed while one of the cornering tests was performed, is shown in Fig. 5.

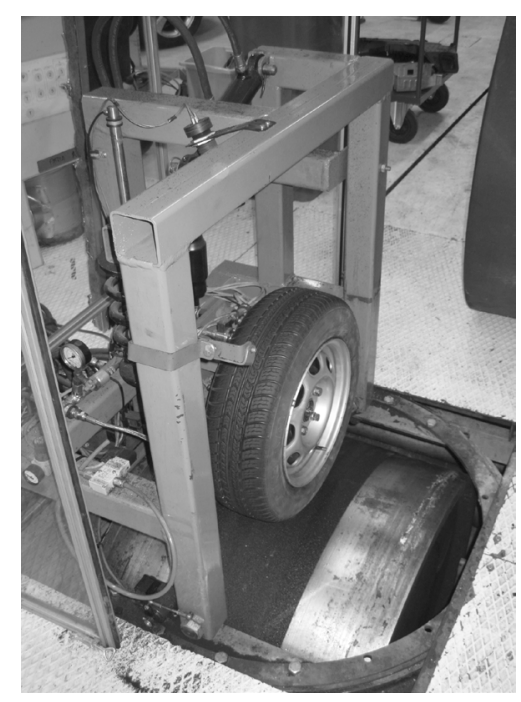

Fig. 4. Test rig used for tire cornering experiments

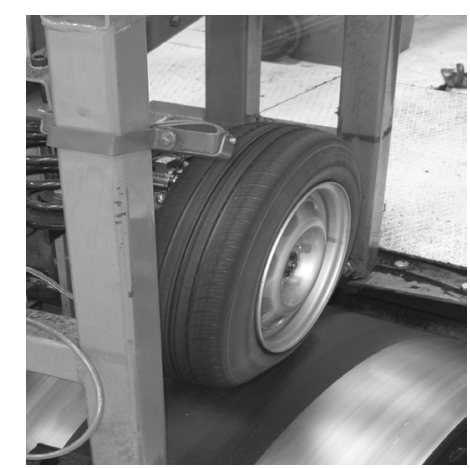

Fig. 5. Tire and drum during cornering test

Two different kinds of tests, with variable operating parameters, have been performed:
1. Braking tests:

- Initial speed: $10 \mathrm{~km} / \mathrm{h}$, wheel loads: $2845 \mathrm{~N}$, $3580 \mathrm{~N}$ and $4287 \mathrm{~N}$.

- Initial speed: $50 \mathrm{~km} / \mathrm{h}$, wheel loads: $2845 \mathrm{~N}$, $3580 \mathrm{~N}$ and $4287 \mathrm{~N}$.

2. Cornering tests, slip angle varying from -10 to $+10^{\circ}$. Inflation pressure has in turn been set to $0.12,0.15,0.2$ and $0.25 \mathrm{MPa}$, wheel load to 2845,3580 and $4287 \mathrm{~N}$, drum speed to 10,50 and $80 \mathrm{~km} / \mathrm{h}$ and camber angle to 0,2 and 4 degrees, thus some 20 different combinations of tire operating parameters have been used.

The operating parameters for cornering tests were chosen appropriately, in order to analyze the influence of wheel load, circumferential drum speed, inflation pressure and camber angle to cornering (side) force and self-aligning torque of free-rolling cornering tire.

Quantities that are directly output by testing rig are: time, $t[\mathrm{~s}]$, slip angle, $\alpha[\mathrm{deg}]$, camber angle, $\gamma$ [deg], dynamic tire radius, $r_{d}$ [mm], temperature $T\left[{ }^{\circ} \mathrm{C}\right]$, circumferential drum speed $v[\mathrm{~km} / \mathrm{h}]$, frequency of wheel rotation, $N$ [1/s], longitudinal force, $F_{x}[\mathrm{~N}]$, lateral force, $F_{y}$ [N], wheel load, $F_{z}[\mathrm{~N}]$, overturning moment, $M_{x}$ $[\mathrm{Nm}]$, rolling resistance moment, $M_{y}[\mathrm{Nm}]$ and self-aligning moment, $M_{z}[\mathrm{Nm}]$.

Quantities that were calculated in order to compare test results to FEA results are:

- $\quad$ angular velocity of the tire, $\omega[\mathrm{rad} / \mathrm{s}]$ :

$$
\omega=2 \pi N,
$$

- $\quad$ effective (rolling) radius, $r_{e}[\mathrm{~mm}]$ :

$$
r_{e}=(277.78 \cdot v) / \omega,
$$

- $\quad$ slip:

$$
\text { slip }=1-((277.78 \cdot v) /(r d \cdot \omega)) .
$$

Test cycles have been adjusted in order to get optimal accuracy of output quantities. An optimal test cycle of a cornering test is shown in Fig. 6.

Cornering test cycle consists of the following intervals: time needed for stabilization of tire rotation, slip angle change from 0 to $+10^{\circ}$, slip angle change from +10 to $-10^{\circ}$, slip angle change from -10 to $0^{\circ}$ and the time needed to stop the rotation. Depending on the ratio of slip angle change, hysteretic effect is more or less visible on the experimental curves that represent the relation 
between slip angle and side force or self-aligning torque. Since experimental results were meant to be compared to the results of quasistatic FEA, the rate of slip angle change has been chosen such as to minimize hysteretic effect (Fig. 7). The vertical force of $3580 \mathrm{~N}$ represents nominal load, force of $4287 \mathrm{~N}$ is equal to the maximum allowable load, while the force of $2845 \mathrm{~N}$ simulates the load less than nominal.

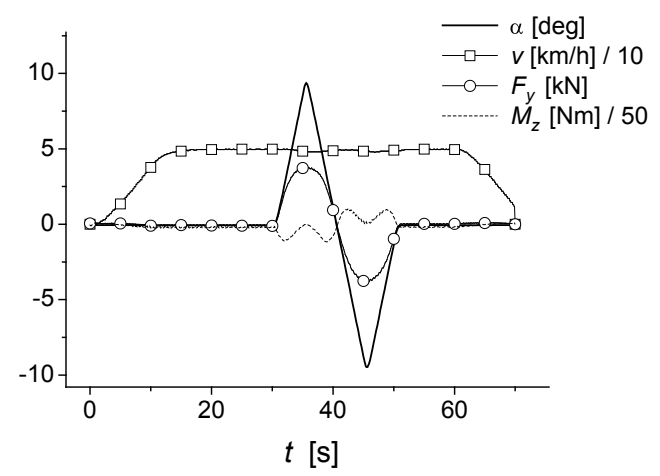

Fig. 6. Cornering test cycle of free-rolling cornering test at circumferential drum speed $v=50 \mathrm{~km} / \mathrm{h}$ and vertical load $F_{z}=3580 \mathrm{~N}$

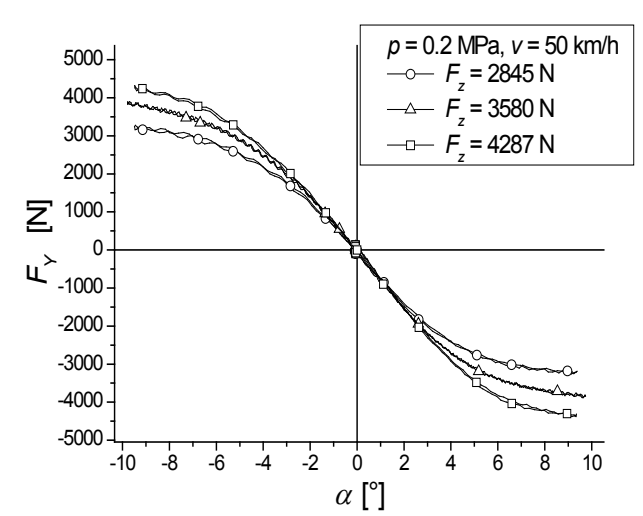

Fig. 7. Experimentally obtained dependency of cornering force on slip angle, at three different values of vertical load

\subsection{Rubber Friction Testing Rig}

In order to find the values of the friction coefficient of tire tread as a function of sliding speed and contact pressure, rubber specimens have been tested using Mini- $\mu-$ road [10] (Fig. 8 ). The testing rig is situated in the cold chamber, where different kinds of arctic weather conditions and sliding on ice may optionally be simulated.
Rubber specimens are usually square-shaped (60 $\times 60 \mathrm{~mm}$ ), but tread blocks, cut from the tire, may also be used.

In this study, tread blocks, cut from another tire, were used for determining the friction coefficient. The other tire was exactly the same as the one that was used on tire testing machine, considering size, type and production date. In such a way, the influence of specimen geometry to test results was meant to be diminished. Double specimens were used to enlarge contact surface and enable testing at lower pressures. On the other hand, to enable testing at higher pressures, single specimens were used (Fig. 9).

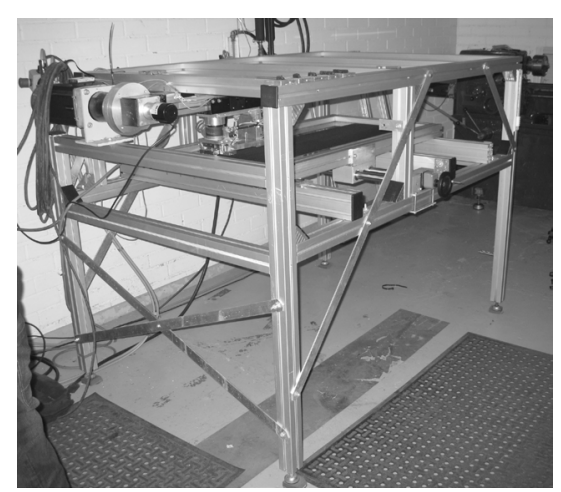

Fig. 8. Mini- $\mu-$ road, linear friction tester that enables sliding of rubber specimens over various kinds of surfaces

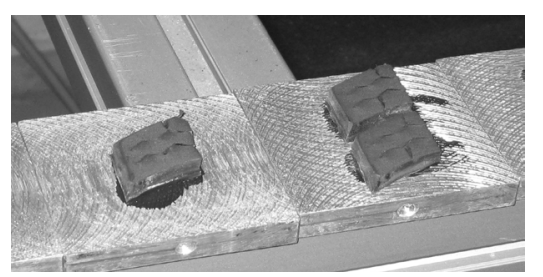

Fig. 9. Single and double specimens have both been used for determination of tire tread friction coefficient

At the beginning of each test cycle, rubber specimen is pressed to the surface (Fig. 10). Pneumatic cylinder that produces normal force as well as force transducer that measures tangential force may be seen in the picture. The required normal force is then calculated in such a way that, when divided by area of specimen contact surface, it produces the desired value of mean contact pressure. While the specimen slides along the surface, at predefined sliding speed, tangential 
force is recorded. Coefficient of friction is then obtained by dividing the mean value of tangential force by the value of normal force.

A measurement has been done in total of 63 cycles, obtained as combinations of 7 different contact pressures and 9 different sliding speeds. Values of friction coefficient obtained by experiment correspond to points shown in Fig. 11. The figure also contains approximating surface, based on rational Chebyshev polynomials. This surface was fitted to experimental points to represent the friction coefficient in given domain of sliding speeds and contact pressures. Based on the surface, a table that contained the values of friction coefficient at equally spaced intervals of sliding speeds and contact pressures was extracted. This table was used to provide input values of tire tread friction within finite element model of the tire.

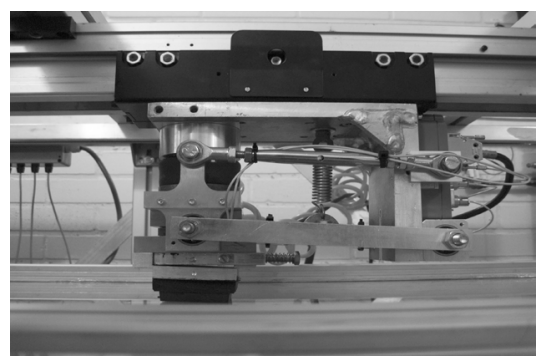

Fig. 10. Close photo of rubber specimen being mounted on the machine and pressed against the

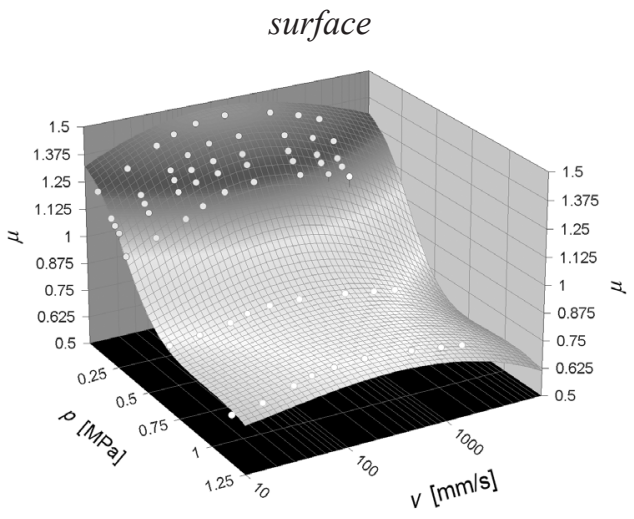

Fig. 11. Friction coefficient of tread rubber $(\mu)$, as a function of sliding speed ( $v)$ and contact pressure ( $p$ )

\section{RESULTS AND DISCUSSION}

The procedure for SSRA of the tire rolling on flat surface has been described in detail in [14].
Model changes, i.e. introduction of a more realistic representation of friction and the replacement of the flat surface with cylindrical one, have contributed to a considerable increase of resulting deformations, strains, stresses and reaction forces comparing to those shown in [14].

Fig. 12 shows FE tire model during simulation of braking.

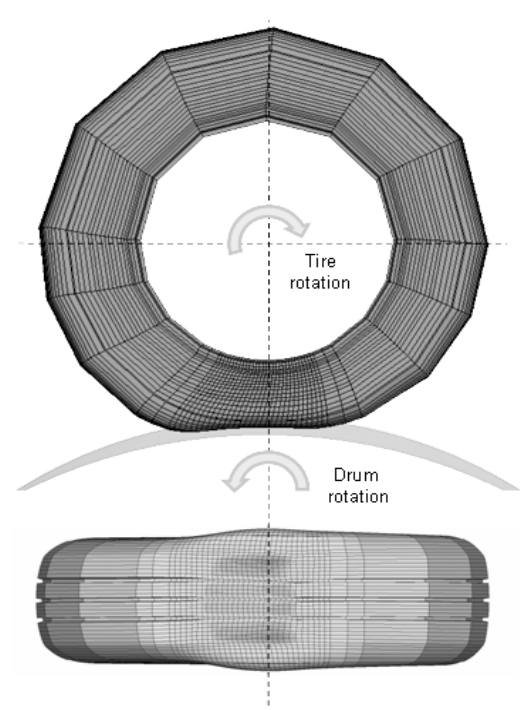

Fig. 12. Deformed shape of the tire model and contact pressure distribution at the footprint during braking analysis

Experimental results related to braking are of lower quality than those related to cornering, as additional sensors of lower resolution had to be used for this kind of test. However, when the experimental parameters were selected so as to minimize measurement errors, the data that could be used for comparison with numerical results, as shown in Fig. 13 were obtained. Lower resolution and accuracy of the measuring device, as well as insufficient strength of drum brake, cause a significant dissipation of experimental data.

Much more attention has been paid to cornering experiments. In this case, experimental results of a considerably better quality than in the case of braking were obtained, as force and moment sensors were not changed. As a substantial number of operating parameters has been varied during cornering experiments, the complete set of experimental results is quite large. 
Thus, only a chosen subset of those will be shown and compared to results of SSRA.

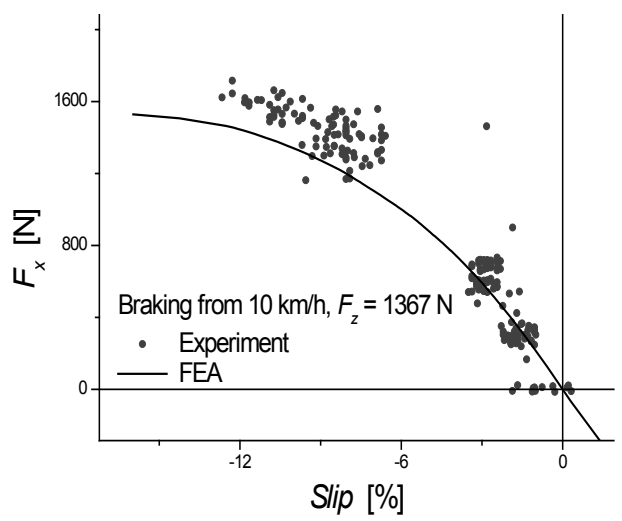

Fig. 13. The comparison of experimentally and numerically obtained correlation of braking force and slip ratio

Fig. 14 shows the deformed shape of tire model during cornering analysis. Very large deformations occur during the analysis, especially at larger values of slip angle. For this reason the FE model should be carefully created. This primarily refers to regularity and size of finite element mesh, choice of material model for all structural components, especially tread and carcass, and the definition of friction between the tread and the ground.

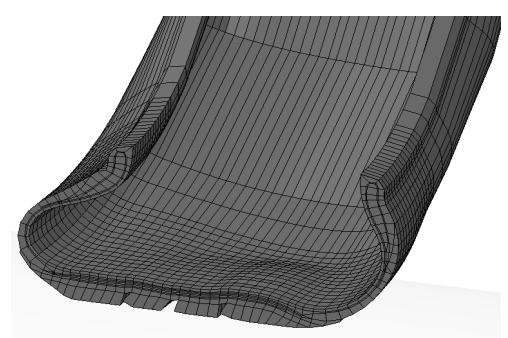

Fig. 14. Detail of deformed tire model during cornering analysis, at slip angle of $10^{\circ}$

Fig. 15 shows contact pressure distribution at the footprint, at different stages of cornering analysis.

Having in mind all the approximations which were necessarily introduced into the analyses, the correlation between experimental and numerical results is considered to be good (Figs. 16 and 17), especially in the case of the right turn (left side of the graphs). Nevertheless, further improvement in accuracy of numerical results was achieved by calibration of friction coefficient, as shown at the end of the paper.

The intensity of residual side force (ply steer) obtained numerically is overestimated, which is considered to be the consequence of insufficient density of belt mesh and the absence of detailed tread on finite element model. It is well known that the tread is usually designed to diminish the ply steer effect. The difference between experimental and numerical results also gets larger for higher values of slip angles.
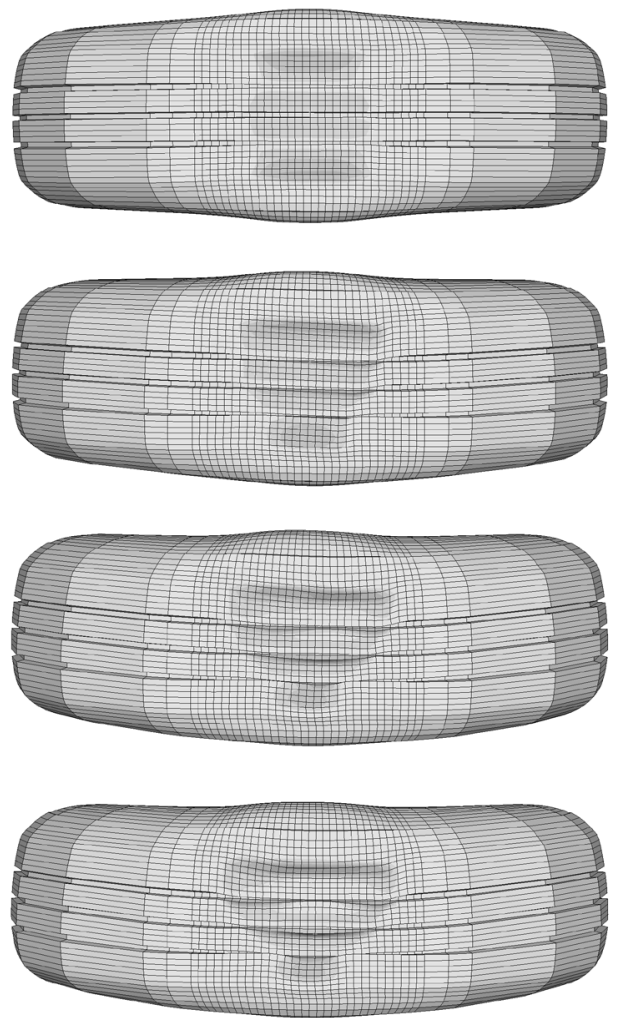

Fig. 15. Footprint shape and contact pressure distribution for tire rolling at $50 \mathrm{~km} / \mathrm{h}$, under nominal vertical load of $3580 \mathrm{~N}$, inflation pressure of $0.2 \mathrm{MPa}$, camber of $0^{\circ}$ and slip angles of $0,3,6$ and $10^{\circ}$

Fig. 18 shows the comparison of slip angle - side force curves obtained numerically for three examined values of vertical load. Their correlation is very similar to the correlation of experimental ones, given in Fig. 7.

Previous figures show the influence of the vertical force on cornering characteristics of the 
tire. Experimental and numerical procedures have also been used for examination of the influence of rolling speed inflation pressure and inclination angle to cornering characteristics.

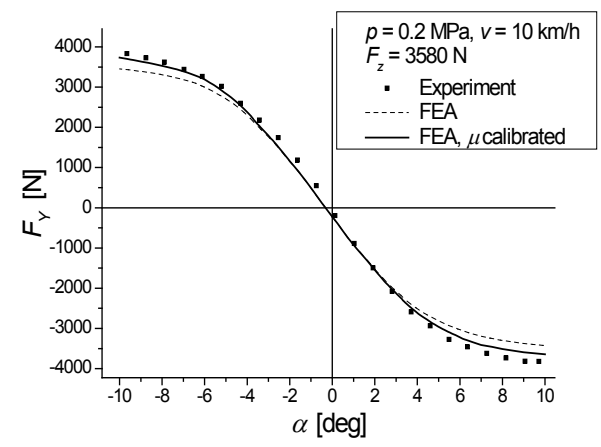

Fig. 16. Comparison of experimental and numerical slip angle-side force curves for the tire inflated to $0.2 \mathrm{MPa}$ rolling at $10 \mathrm{~km} / \mathrm{h}$ at vertical load of $3580 \mathrm{~N}$

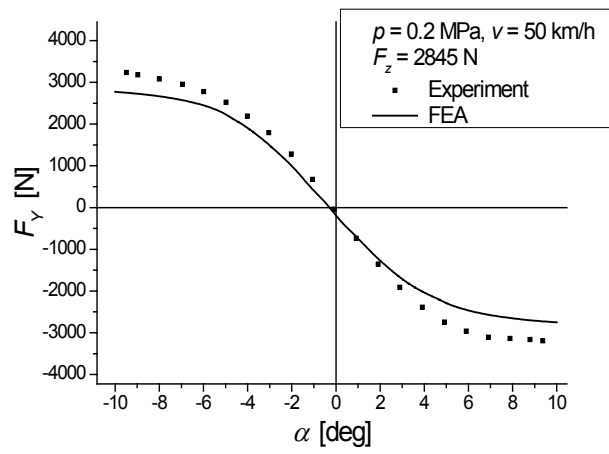

Fig. 17. Comparison of experimental and numerical slip angle-side force curves for the tire inflated to $0.2 \mathrm{MPa}$ rolling at $50 \mathrm{~km} / \mathrm{h}$ at vertical load of $2845 \mathrm{~N}$

The comparison between numerical and experimental results showed that the intensity of numerically determined forces always had the tendency to be lower, the difference being between 10 and $15 \%$. The difference was also becoming more significant at higher values of the slip angle. Thus, the room for improvement in numerical results was spotted.

At higher slip angles, a large percentage of tread surface is slipping and thus the friction at the footprint plays the most important role in generation of tire forces and moments. For that reason, the approach to friction modeling was once more reconsidered. At first, different fits to experimental friction data were used as an alternative to original one, but no significant change in numerical results was noticed. Then, the experiment used to obtain friction data was simulated using FEA (Fig. 19), in order to determine how the nature of the test itself influences the resulting values of friction coefficient. The data on friction coefficient obtained by surface fitting to experimental points, as described earlier, were used as an input.

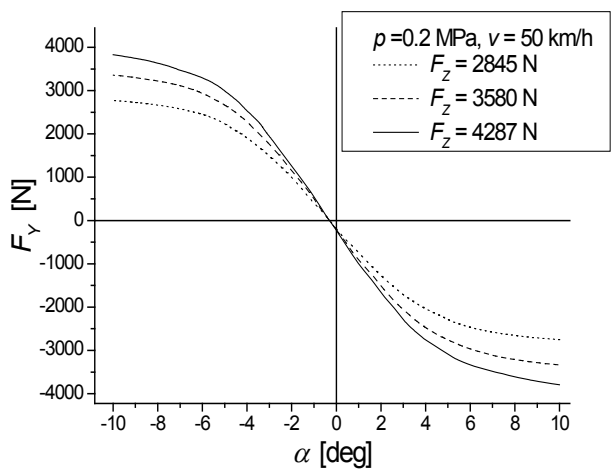

Fig. 18. Comparison of numerical slip angleside force curves for the tire inflated to $0.2 \mathrm{MPa}$ rolling at $50 \mathrm{~km} / \mathrm{h}$ at three different values of vertical load

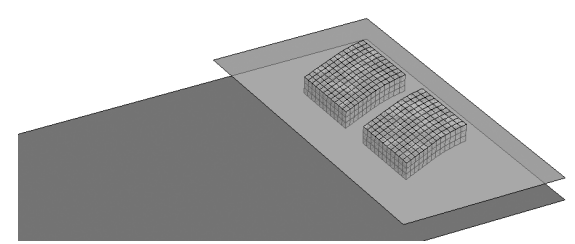

Fig. 19. FE model for simulation of experimental determination of tire tread friction coefficient

The simulations showed that there exists a large inhomogenity of contact pressure at specimen surface and that the pressure is much higher at leading edge of the specimen (Fig. 20). This phenomenon is considered to be the cause of noticeable differences in actual and predicted coefficient of friction. Thus, a calibrating function was introduced, by which the originally fitted friction surface was multiplied in order to obtain the corrected one, similar to the approach described in [15]. Fig. 21 illustrates this procedure at mean contact pressure of 0.11 $\mathrm{MPa}$. When the corrected coefficient of friction (curve Fit corrected) was used in the simulation of the experiment, the results were obtained that 
correspond to experimental ones much better (curve FEA-corrected).

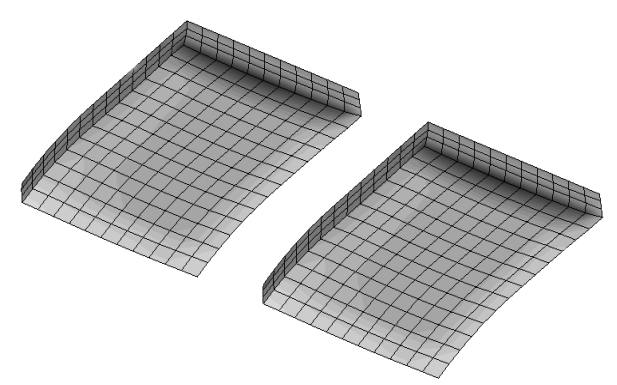

Fig. 20. Contact pressure distribution at the surface of double tire tread specimen sliding on flat surface obtained by FEA, at mean contact pressure of $0.11 \mathrm{MPa}$ and sliding speed of 10 $\mathrm{mm} / \mathrm{s}$

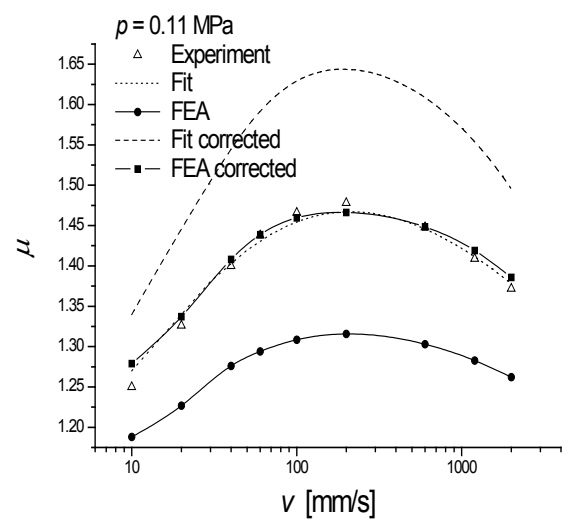

Fig. 21. Calibration of friction coefficient, shown at contact pressure of $0.11 \mathrm{MPa}$

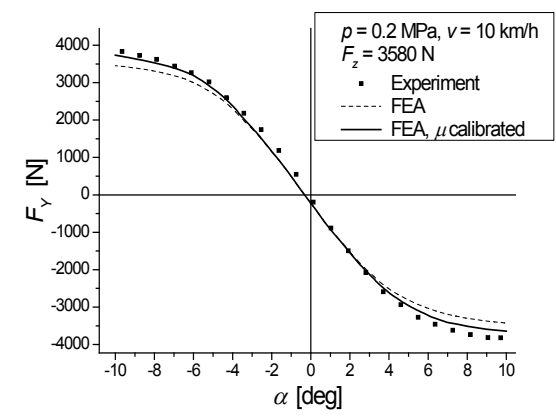

Fig. 22. Comparison of experimental and numerical slip angle - side force curves for the tire inflated to $0.2 \mathrm{MPa}$ rolling at $10 \mathrm{~km} / \mathrm{h}$ at vertical load of $3580 \mathrm{~N}$, calibrated vs. noncalibrated coefficient of friction

In order to test the effectiveness of the calibration process, the calibrated friction coefficient has been used in a couple of repeated tire cornering simulations. The results of those are shown in Figs. 22 and 23. When the calibrated results were used, the difference between experimental and numerical results was noticeably smaller, reaching a maximum of $5 \%$.

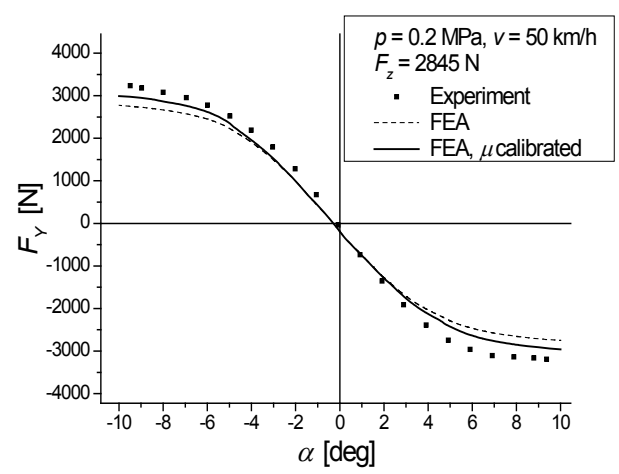

Fig. 23. Comparison of experimental and numerical slip angle - side force curves for the tire inflated to $0.2 \mathrm{MPa}$ rolling at $50 \mathrm{~km} / \mathrm{h}$ at vertical load of $2845 \mathrm{~N}$, calibrated vs. noncalibrated coefficient of friction

\section{CONCLUDING REMARKS}

The finite element model for FEA of tire rolling on the drum has been presented, which demonstrates the flexibility of CAD based meshing approach introduced by the authors. The results of the analyses conducted on the model have successfully been compared to experimental ones, confirming FE model validity. The differences between experimental and numerical results were decreased after the calibration of friction coefficient had been performed. In such a way the further improvement of the existing model was achieved.

The presented FE tire model and associated analyses are used for performing parametric studies within the tire design process, helping the tire designer to quickly find the optimal values of tire design parameters. The tire design process is thus shortened and at the same time greater predictability and improvement of tire performance are achieved.

In order to further improve the existing model, future activities are planned. On the one hand, the work on the improvement in modeling of tire tread friction will be undertaken. On 
the other, the FE tire model will be modified to include the representation of detailed tire tread, by enhancing the CAD based meshing approach to $3 \mathrm{D}$ geometry.

\section{AKNOWLEDGMENT}

The authors would like to express their gratitude to Mr. Panu Sainio and Research Group for Vehicle Engineering, School of Engineering, AALTO-University (formerly Helsinki University of Technology, TKK), for their invaluable help in tire and friction testing.

\section{REFERENCES}

[1] Tönük, E., Ünlüsoy, S. (2001). Prediction of automobile tire cornering force characteristics by finite element modeling and analysis. Computers and Structures, vol. 79, no. 13 , p. 1219-1232, DOI:10.1016/S00457949(01)00022-0.

[2] Kabe, K., Koishi, M. (2000). Tire cornering simulation using finite element analysis. Journal of Applied Polymer Science, vol. 78, no. 8, p. 1566-1572, DOI:10.1002/10974628(20001121)78:8<1566::AID-APP140> 3.0.CO;2-I.

[3] Ghoreishy, M.H.R. (2006). Steady state rolling analysis of radial tire: comparison with experimental results. Proceedings of the Institution of Mechanical Engineers, Part D: Journal of Automobile Engineering, vol. 220, no. 6, p. 713-721, DOI:10.1243/09544070JAUTO268.

[4] Olatunbosun, O.A., Bolarinwa, O. (2004). FE simulation of the effect of tire design parameters on lateral forces and moments. Tire Science and Technology, TSTCA, vol. 32, no. 3, p. 146-163, DOI:10.2346/1.2186779.

[5] Dorsch, V., Becker, A., Vossen, L. (2002). Enhanced rubber friction model for finite element simulations of rolling tyres. Plastics, Rubber and Composites, vol. 31, no. 10, p. 458464, DOI:10.1179/146580102225006486.

[6] Pottinger, M.G. (2005). Forces and Moments. Gent, A.N., Walter, J.D. (Eds.), The pneumatic tire. National Highway Traffic
Safety Administration, U.S. department of Transportation, Washington D.C., p. 286-363.

[7] Helsinki University of Technology, Laboratory of Automotive Engineering, Dynamic tyre force measuring device, from http://edp.tkk.fi/en/research/research facilities/tkk_dynamic_tyre_force_measuring_device.pdf, accessed on 2009-09-05.

[8] Guo, K.H., Zhuang, Y., Chen, S.K., Willlam L. (2006). Experimental research on friction of vehicle tire rubber. Frontiers of Mechanical Engineering in China, vol. 1, no. 1, p. 14-20, DOI:10.1007/s11465-005-0001-z.

[9] Božić, A., Petrović, I., Matuško, J. (2009). Experimental investigations and modeling of the rubber-asphalt sliding pair dynamics. Strojniški vestnik - Journal of Mechanical Engineering, vol. 55, no. 5, p. 303-318.

[10] Helsinki University of Technology, Laboratory of Automotive Engineering, Mini- $\mu-$ road, Rubber Friction Measuring Device, from http://edp.tkk.fi/en/research /research_facilities/tkk_mini-m-road.pdf, accessed on 2009-09-05.

[11] Stojković, M., Manić, M., Trajanović, M. (2005). Knowledge-embedded template concept. CIRP - Journal of Manufacturing Systems, vol. 34, no. 1, p. 71-79.

[12] Korunović, N., Trajanović, M., Manić, D., Manić, M. (2004). Rubber modeling for tire FEA. World of Polymers, vol. 7, no. 3, p. 8594. (In Serbian)

[13] Korunović, N., Trajanović, M., Stojković, M. (2007). FEA of tires subjected to static loading. Journal of Serbian Society for Computational Mechanics, vol. 1, no. 1, p. 87-98.

[14] Korunović, N., Trajanović, M., Stojković, M. (2008). Finite element model for steadystate rolling tire analysis. Journal of Serbian Society for Computational Mechanics, vol. 2, no. 1, p. 63-79.

[15]Huemer, T., Liu, W.N., Eberhardsteiner, J., Mang, H.A. (2001). A 3D finite element formulation describing the frictional behavior of rubber on ice and concrete surfaces. Engineering Computations, vol. 18, no. 3-4, p. 417-437, DOI:10.1108/02644400110387109. 